\title{
NOUVELLe
}

\section{Rôle crucial des corécepteurs CD4 et CD8 dans la reconnaissance antigénique des lymphocytes $T \alpha \beta$}

François Van Laethem, Ingrid Saba, Anastasia N. Tikhonova, Alfred Singer
Experimental Immunology Branch, National Cancer Institute,

Bethesda,

MD, États-Unis.

vanlaetf@mail.nih.gov
$>$ Le système immunitaire permet au corps de se défendre contre les bactéries et virus. Ce système permet également d'éliminer les cellules anormales ou cancéreuses qui peuvent se développer. Les lymphocytes B et $T$ sont les principales armes dont dispose le corps pour se débarrasser des corps étrangers ou des cellules anormales. Les cellules B produisent des immunoglobulines ou anticorps, et les cellules $T$ expriment les récepteurs TCR ( $T$ cell receptor) à leur surface. La majorité des lymphocytes $T$ expriment un TCR $\alpha \beta$ alors qu'une minorité expriment un TCR $\gamma \delta$. Les récepteurs des cellules $T$ et $B$ partagent la même propriété de reconnaissance antigénique et sont capables de provoquer une réponse immunitaire. Même si les deux types de lymphocytes utilisent les mêmes mécanismes enzymatiques (les protéines de recombinaison Rag) pour générer leurs récepteurs $B C R$ et $T C R$, et donc la diversité de leur répertoire, leur mode de reconnaissance de l'antigène est très différent.

\section{Mode de reconnaissance antigénique}

Les anticorps (immunoglobulines) produits par les cellules $B$ reconnaissent des antigènes natifs, libres dans la circulation et qui ne requièrent pas de cellules présentatrices d'antigènes (APC). Le TCR $\alpha \beta$ est, quant à lui, incapable de reconnaître des antigènes sous leur forme soluble; ceux-ci doivent être «présentés» sous la forme de peptides associés à des molécules du CMH (complexe majeur d'histocompatibilité) qui servent donc de présentoir pour ces antigènes. Toutes les cellules nucléées expriment les molécules CMH de classe I présentant des peptides issus de protéines cytosoliques, qui seront reconnus par les lymphocytes cytotoxiques $C D 8^{+}$. Le CMH de classe II, quant à lui, est exprimé uniquement par les cellules présentatrices d'antigènes professionnelles comme les macrophages, les cellules dendritiques et les lymphocytes B, et il présente des peptides «étrangers » aux lymphocytes auxiliaires $\mathrm{CD}^{+}$. Cette nécessité absolue d'une liaison des peptides au $\mathrm{CMH}$ pour leur reconnaissance par les lymphocytes TCR $\alpha \beta$ distingue ces derniers de tous les autres types cellulaires du système immunitaire, et permet à ces cellules de jouer leurs rôles cytotoxique ou auxiliaire. Comprendre comment le système immunitaire permet le développement de cellules T $\alpha \beta$ dont les TCR ne voient que le complexe peptide/CMH et non des antigènes natifs (restriction $\mathrm{CMH}$ ) est donc une question fondamentale en immunologie et qui continue de fasciner.

La restriction vis-à-vis du CMH : une propriété génétiquement prédéterminée ou acquise lors de la sélection thymique?

Deux théories ont été émises pour expliquer la restriction de reconnaissance des lymphocytes T $\alpha \beta$ vis-à-vis du CMH.

La première, proposée par les groupes de K.C. Garcia et P. Marrack, postule que des éléments génétiquement non variables du TCR (boucles appelées les régions
CDR ou complementary diversity region) sont «programmées » pour reconnaître le $\mathrm{CMH}[1,2]$. En effet, des études tridimensionnelles par cristallographie ont montré que les TCR adoptent généralement la même orientation diagonale par rapport aux hélices du CMH. Les boucles hypervariables CDRl et 2, qui demeurent intactes lors de l'étape de synthèse du $T C R$, interagissent avec les hélices du $\mathrm{CMH}$, alors que la boucle hypervariable CDR3, qui est modifiée lors de l'assemblage du TCR, interagit avec le peptide à cause de sa grande diversité. En étudiant une famille de chaînes TCR $\beta$ chez la souris, ces équipes de chercheurs ont montré que quelques résidus dans les boucles CDRl et 2 étaient très conservés au cours de l'évolution. En effet, grâce à des expériences de mutagenèse, ces auteurs ont montré que ces acides aminés, qui sont en contact direct avec les hélices $\mathrm{du} \mathrm{CMH}$, sont indispensables pour une sélection thymique optimale [3].

La seconde hypothèse que nous avons avancée pour expliquer l'obsession $\mathrm{du}$ TCR $\alpha \beta$ pour les molécules du CMH, repose entièrement sur la signalisation du TCR dans le thymus [4]. Le TCR qui engage un ligand ne peut, à lui seul, déclencher une signalisation. En réponse à l'interaction entre les TCR et leurs ligands spécifiques, des résidus tyrosine contenus dans les régions cytoplasmiques des chaînes $\operatorname{CD} 3 \varepsilon$ et $z$ nommées ITAM (immunoreceptor-based activation motif) sont phosphorylés. Cette phosphorylation est orchestrée par deux membres de la famille de protéines 


\section{Sélection thymique}

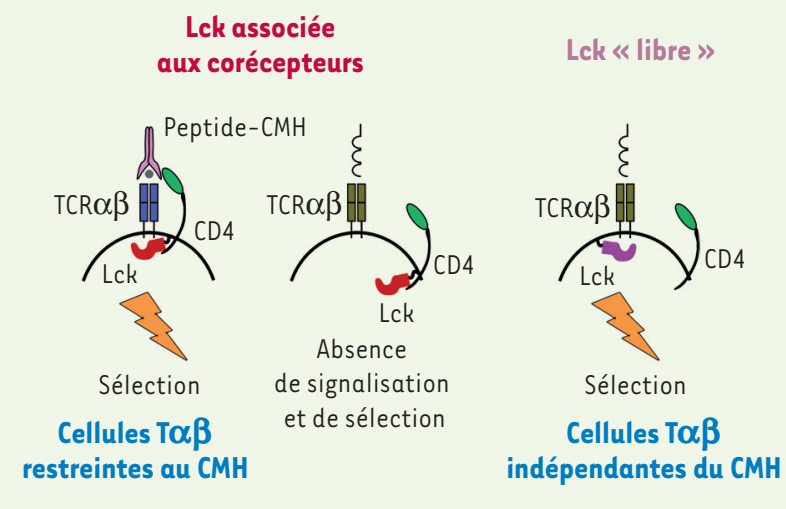

Répertoire TCR $\alpha \beta$

Figure 1. Rôle de la Lck dans la sélection du répertoire des cellules $T \boldsymbol{\alpha} \boldsymbol{\beta}$. La kinase Lck est associée aux domaines cytoplasmiques des corécepteurs CD4 et CD8 dans les thymocytes immatures $C D 4^{+} C D 8^{+}$. Le seul moyen d'obtenir un signal dépendant du CMH est d'engager un des corécepteurs avec le TCR afin d'y amener Lck. Des TCR qui reconnaissent des ligands indépendamment du $\mathrm{CMH}$ sont incapables de recevoir un signal car Lck ne sera pas dans le complexe TCR. Le seul moyen d'obtenir des TCR ayant des spécificités indépendantes du CMH est de «libérer » Lck afin de faciliter la signalisation de tels récepteurs.

qui impose la sélection d'un répertoire des lymphocytes T $\alpha \beta$ restreint kinases Src, Lck et Fyn, exprimés par les lymphocytes T, Lck jouant un rôle prédominant. Cette première étape de phosphorylation induit une cascade de signalisation qui aboutit à l'activation et la sélection des thymocytes. Lck est exprimée tout au long de la vie d'une cellule $T$ et des modifications lipidiques (myristoylation et palmitoylation) lui assurent un ancrage membranaire. Au stade initial du développement thymique (les cellules sont à un stade auquel ni CD4 ni CD8 ne sont exprimés, stade dit double négatif $\left(D 4^{-}\left(D 8^{-}\right)\right.$, Lck n'est pas ancrée à la membrane et est donc «libre ». Ceci est essentiel pour assurer la signalisation du pré-TCR. Ce signal va induire une forte prolifération, suivie de l'expression des corécepteurs CD4 et CD8 pour atteindre le stade de cellule double positive $\operatorname{CD} 4^{+} \mathrm{CD} 8^{+}$: à ce stade, les deux chaînes du TCR sont enfin exprimées pour former un TCR $\alpha \beta$ mature qui peut lier les ligands exprimés à la surface des cellules thymiques épithéliales. À ce stade, toutes les molécules Lck sont associées aux corécepteurs CD4 et CD8. Le nombre de ces corécepteurs exprimés à ce stade double positif étant très élevé, Lck se trouve donc « séquestrée » par les portions cytoplasmiques des corécepteurs. L'affinité des corécepteurs pour les molécules du $\mathrm{CMH}$ est très grande et nous pensons que c'est cette séquestration par les corécepteurs uniquement au CMH. En effet, le seul moyen pour les TCR de recevoir un signal dépendant de Lck est d'engager un complexe peptide/CMH en association avec un des corécepteurs. En effet, même si le TCR est capable de se lier à un ligand autre qu'un complexe peptide/CMH, la séquestration de Lck par les corécepteurs empêchera toute induction de signalisation (Figure l).

\section{La disponibilité de Lck impose la restriction au $\mathrm{CMH}$}

Afin de tester cette hypothèse de séquestration de Lck par les corécepteurs CD4 et CD8, notre laboratoire a génétiquement modifié l'association de Lck avec les corécepteurs [5]. Dans un premier temps, nous avons utilisé un modèle de souris génétiquement déficientes pour les deux corécepteurs CD4 et CD8 ainsi que pour les molécules de $\mathrm{CMH}$ de classe I et II, souris que nous avons appelées quadruple-déficientes (Quad). Dans les thymocytes Quad, Lck est constamment sous forme libre et n'est pas liée aux corécepteurs puisqu'ils sont absents. À l'aide du modèle Quad, nous avons démontré que Lck pouvait s'associer au TCR en l'absence des corécepteurs. La réexpression d'un transgène codant pour CD4 était suffisante pour empêcher cette association entre le TCR et Lck. Malgré l'absence de corécepteurs et du CMH, ces souris ont un nombre important de cellules T $\alpha \beta$. Afin d'étudier la spécificité de ces TCR, nous avons effectué des expériences in vitro de MLR (mixed leukocyte reaction). Contrairement aux cellules T $\alpha \beta$ provenant de souris sauvages, qui ne prolifèrent qu'en présence d'APC allogéniques (exprimant un $\mathrm{CMH}$ différent des leurs), les cellules $T \alpha \beta$ des souris Quad prolifèrent en présence d'APC qu'elle qu'en soit l'origine, y compris celles de souris déficientes pour les molécules de CMH. Ces expériences ont donc montré pour la première fois qu'en l'absence de séquestration de Lck par les corécepteurs, le répertoire T $\alpha \beta$ est élargi et peut contenir des TCR qui reconnaissent des antigènes qui ne sont pas liés au CMH. Dans un deuxième temps, nous avons créé une souris transgénique qui contient une mutation des deux résidus cystéine indispensables à la liaison de Lck aux corécepteurs. Ces souris expriment les corécepteurs et les molécules du CMH ainsi qu'une forme mutée de Lck. Les souris exprimant la forme mutée de Lck ont le même phénotype que celui décrit pour les souris Quad.

\section{Identification d'un ligand pour des TCR indépendants du CMH}

Afin de caractériser la spécificité unique des TCR exprimés dans les souris Quad, nous avons isolé les cellules $T$ de ces souris et généré des hybridomes afin d'analyser la spécificité du TCR. Nous avons découvert qu'un certain nombre d'hybridomes reconnaissent le même antigène, c'est-à-dire la protéine de 
surface CD155 [6]. La molécule murine CD155 est l'homologue du récepteur humain du virus de la polio, et elle est exprimée par la plupart des cellules hématopoïétiques. In vitro, nous avons montré que deux TCR de souris Quad reconnaissent CD155 en l'absence d'APC et avec une affinité dix fois supérieure à celle des TCR restreints au CMH. Nous avons créé des souris transgéniques exprimant deux récepteurs spécifiques pour CD155. Ces deux TCR sont capables d'induire des signaux en l'absence de $\mathrm{CMH}$ et de corécepteurs in vivo, contrairement aux TCR conventionnels qui reconnaissent le complexe peptide/CMH. Malgré les études caractérisant les peptides périphériques capables de stimuler des TCR restreints au $\mathrm{CMH}$, les peptides capables d'induire la sélection positive dans le thymus ne sont pas encore connus. En croisant les souris transgéniques exprimant un TCR spécifique pour CD155, et les souris déficientes pour CD155, nous avons pu montrer pour la première fois que le ligand indépendant du CMH qui stimule les cellules en périphérie est également indispensable pour la sélection positive dans le thymus [7]. En présence de CD155, un très grand nombre de cellules $T \alpha \beta$ transgéniques se développent, nombre qui est réduit à néant quand les souris sont déficientes pour CD155.
Importance des résidus conservés du CDR2 pour la sélection thymique Un des TCR spécifique que nous avons caractérisé et qui est spécifique de CD155 contient également des résidus dans la boucle CDR2 qui sont indispensables à la reconnaissance du CMH et la sélection thymique tel que prédit par les groupes de Garcia et Marrack. In vivo, nous avons montré que ces mêmes résidus étaient également indispensables pour la sélection thymique indépendante du CMH par CDI55 [7].

\section{Conclusion}

Nos récents travaux ont montré que l'association de Lck avec les corécepteurs CD4 et CD8 est indispensable pour générer un répertoire de cellules T $\alpha \beta$ restreint aux peptides présentés par le CMH. En effet, l'engagement d'un des corécepteurs est le seul moyen pour les TCR qui sont restreints au $\mathrm{CMH}$ d'avoir accès à la protéine Lck nécessaire à la sélection thymique. De plus, l'idée qui avait été émise et selon laquelle des résidus conservés de la boucle CDR2 prédisposeraient le TCR à reconnaître uniquement le $\mathrm{CMH}$ ne semble pas se vérifier complètement, car ces mêmes résidus sont également très importants pour la reconnaissance antigénique indépendamment du CMH. Nous pensons que ces TCR indépendants du $\mathrm{CMH}$ ont un potentiel théra- peutique. En effet, ils peuvent être utilisés pour reconnaître et déclencher une réponse contre un antigène tumoral car, contrairement aux TCR «classiques », ils ont une affinité très élevée et ne sont pas dépendants de la compatibilité au CMH. $\diamond$ Cruciall role of CD4 and CD8 coreceptors in antigen recognition of $\alpha \beta$ T lymphocytes

\section{LIENS D'INTÉRÊT}

Les auteurs déclarent n'avoir aucun lien d'intérêt concernant les données publiées dans cet article.

\section{RÉFÉRENCES}

1. Garcia KC. Reconciling views on T cell receptor germline bias for MHC. Trends Immunol 2012 ; 33 : 429-36.

2. Marrack P, Scott-Browne JP, Dai S, et al. Evolutionarily conserved amino acids that control TCR-MHC interaction. Annu Rev Immunol 2008 ; 26 : 171-203.

3. Scott-Browne JP, White J, Kappler JW, et al. Germlineencoded amino acids in the alphabeta T-cell receptor control thymic selection. Nature 2009 ; 458 : 1043-6.

4. Van Laethem F, Tikhonova AN, Singer A. MHC restriction is imposed on a diverse $T$ cell receptor repertoire by $\mathrm{CD} 4$ and $\mathrm{CD} 8$ co-receptors during thymic selection. Trends Immunol 2012 ; 33 : 437-41.

5. Van Laethem F, Sarafova SD, Park JH, et al. Deletion of CD4 and CD8 coreceptors permits generation of alphabeta $T$ cells that recognize antigens independently of the MHC. Immunity $2007 ; 27$ : 735 50.

6. Tikhonova AN, Van Laethem F, Hanada $K$, et al. alphabeta $T$ cell receptors that do not undergo major histocompatibility complex-specific thymic selection possess antibody-like recognition specificities. Immunity 2012; $36: 79-91$.

7. Van Laethem F, Tikhonova AN, Pobezinsky LA, et al. Lck availability during thymic selection determines the recognition specificity of the T cell repertoire. Cell $2013 ; 154: 1326-41$.

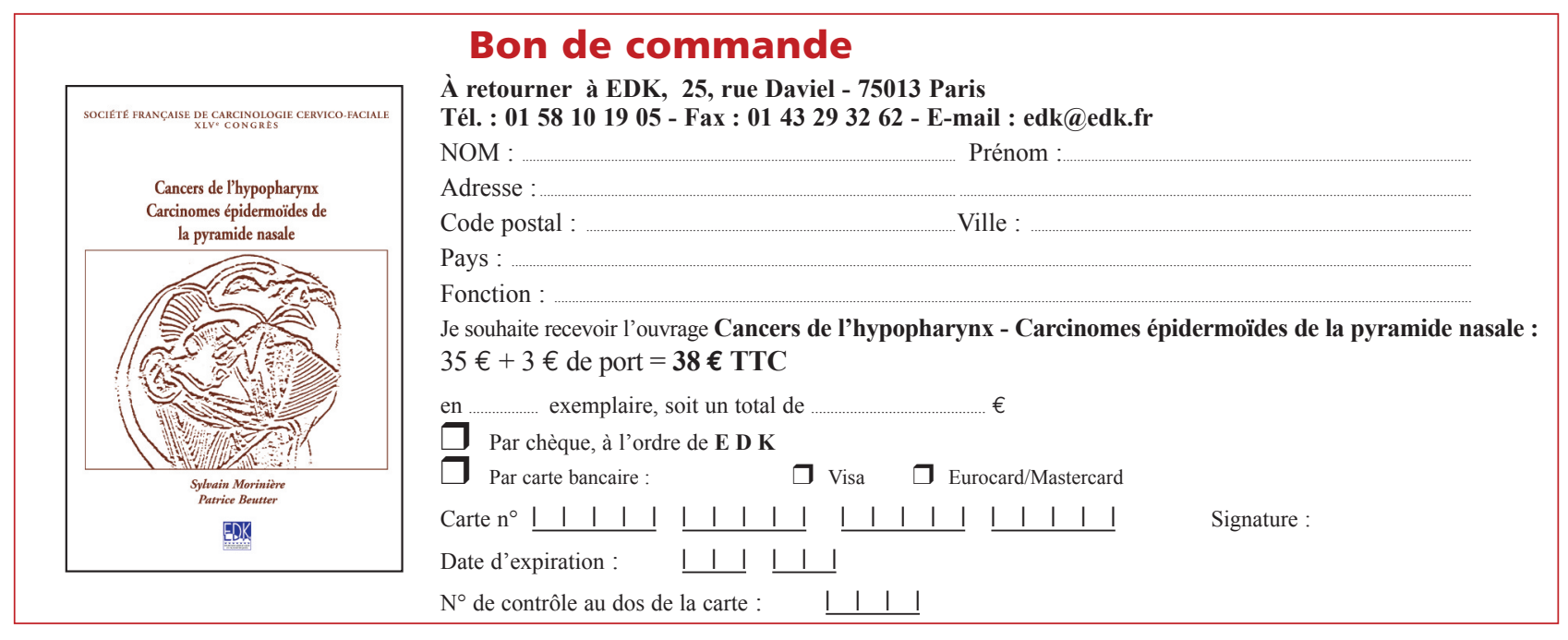

